\title{
FORCE FINDING OF SUSPENDED-DOMES USING BACK PROPAGATION (BP) ALGORITHM
}

\author{
Jiamin Guo ${ }^{1, *}$, Xingfei Yuan ${ }^{2}$, Zhixin Xiong ${ }^{1}$ and Shilin Dong ${ }^{2}$ \\ ${ }^{1}$ School of Ocean Science and Engineering, Shanghai Maritime University, Shanghai 200135, China \\ ${ }^{2}$ Department of Civil Engineering, Zhejiang University, Hangzhou 310058, China \\ *(Corresponding author: E-mail: guojiamin@zju.edu.cn)
}

Received: 2 April 2014; Revised: 4 February 2015; Accepted: 23 February 2015

\begin{abstract}
Force finding is a key step when designing a suspended-dome. To give a general simple method for force finding, this report accomplished research on the application of a BP network to force finding of a suspended-dome under a certain tension process. First, this paper gives three typical states of the construction process, and then, it selects the internal force in the hoop cables at the initial state as the input data and the initial strain of the active element at the zero state as the output data. Then, a three-layer BP network was developed for force finding of the suspended-dome model. Second, this paper presents a method for restricting the training set range, in which 1000 sets of patterns were generated by the finite element software ANSYS. Then, we randomly selected 19 groups of training data from 1000 sets of samples to train the BP network and predicted the results of force finding. Last, this paper combines GA with BP to predict the results of force finding. The results show that the BP network can solve the force-finding problem accurately and effectively when the training samples are sufficient. The prediction stability of the BP network and the prediction precision can be significantly enhanced after the initial weights and the thresholds are optimized by a GA.
\end{abstract}

Keywords: Suspended-dome, BP network, Finite element, initial strain, force-finding

DOI: $10.18057 /$ IJASC.2016.12.1.2

\section{INTRODUCTION}

Tensegrity structures, which consist of continuous tension elements and discontinuous compression elements, were proposed by Fuller, and the first tensegrity structure was designed by Snelson in 1948. To date, the tensegrity concept has become a basic principle of nature and has been applied to so many fields of science that perhaps it is losing its main meaning (Gómez [1]). With respect to the basis of the tensegrity concept, Geiger invented the cable dome, which includes a compressed ring in the boundary of a tensegrity structure (Pellegrino [2]). Since then, the first cable dome was designed by Geiger: for the Olympics in Seoul (1986), followed by the Redbrid Arena in Illinois (1988), the Florida Suncoast Dome in St. Petersburg (1988), and the Taoyuan Arena in Taiwan (1993). At the same time, its large hoop beam and its difficult construction has constrained its widespread application. In 1993, Kawaguchi et al[3] introduced the single-layer latticed shell into the cable dome and invented the suspended-dome, which take advantage of both structural systems (Chen et al. [4]). As an improved cable dome, suspended-dome replaces the upper cables of the cable dome with a single-layer latticed shell that can provide a certain initial stiffness, and as a result, its construction is simplified. Suspended-dome is also an improved single-layer latticed shell with a lower cable-strut, which can strengthen the upper shell.

Suspended-dome consists of the compression-only vertical struts, the tension-only hoop cables and radial cables. Since it was proposed, suspended-dome has been attracting much attention from both researchers and engineers. These investigators have performed some theoretical and experimental research on the structural mechanical properties, including construction simulation and morphological analysis. 
Kang and Chen [5] noted that the lower cable-strut could reduce the internal force of a single-layer latticed shell. Additionally, model tests on a small-scale suspended-dome indicated that the stability and the rigidity have been improved greatly compared with the single-layer latticed shell (Kawaguchi et al. [6]). In addition, The internal force flow in suspended-dome could be built in a closed loop, thus making the structure a self-equilibrated system, and a weak boundary bearing system becomes possible (Zhang et al. [7]). Dong et al.[8] studied tension schemes by an experimental model and noted that the construction difficulty is lower than the cable domes and that tensioning radial cables is better than tensioning hoop cables. These favorable properties make suspended-dome an ideal choice for many applications. In Japan, the Hikarigaoka Dome and the Fureai Dome have been constructed. In China, suspended-dome was employed in structures for the Olympics in Beijing (2008), the Olympic Sports Centre's Gymnasium in Changzhou, and the Olympic Centre in Jinan (2009).

In practical design and analysis, the determination of the initial configuration and the initial prestress is of great importance for suspended-dome structures; this analysis approach is known as morphological analysis. Much research on the morphology of tensegrity structures has been performed[9, 10]. However, few studies have been performed for suspended-dome structures. A simplified strategy for force finding analysis of suspended-domes based on the local analysis method was present (Cao and Zhang [11]), but the present strategy did not consider the influence of the actual construction process. Zhuo et al. [12] studied the control algorithm of tension force on the basis of the measured internal forces in the actual construction and gave the tension force correction calculation method. However, the developed methods appear to be inconvenient for engineers to operate. Based on the known parameters, the morphological analysis of the suspended-dome was classified into three categories: form finding, force finding and form and force finding, but they cannot be described by an algorithm that is based on mathematical concepts (Guo et al [13]).

The back propagation algorithm (BP) has a vigorous ability to solve problems that cannot be described in terms of a mathematical concept. After McCulloch and Pitts (1943) introduced models of neurological networks, Rumelhart and McClelland developed the back-propagation of error learning procedure (abbreviated: BP), and realized the concept that non-linearly separable problems could be solved by multilayer perception. From that time on, the multi-layered network trained by the back-propagation algorithm has been applied extensively to solve various engineering problems $[14,15]$. In the following, this paper will focus on the force finding of a suspended-dome under conditions of different tension processes by using a BP neural network.

\section{FORCE FINDING}

During the overall construction, suspended-dome will experience three typical states: the zero state, initial state and loading state [16]. The structure will include different loads under different states. This paper selects the initial strain to simulate pre-stress on a numerical model. For practical projects and numerical models, the state parameters and the loads that were considered under different states are described in detail in Tables 1-2. 
Table 1. Three Typical States and State Parameters for Practical Projects

\begin{tabular}{lccccc}
\hline Typical & \multicolumn{3}{c}{ Loads considered } & \multicolumn{2}{c}{ State parameters } \\
\cline { 2 - 6 } states & pre-stress & self-weight & outer loads & geometry & force \\
\hline Zero state $(Z)$ & $\times$ & $\times$ & $\times$ & $G_{Z}$ & $F_{Z}$ \\
Initial state $(I)$ & $\sqrt{ }$ & $\sqrt{ }$ & $\times$ & $G_{I}$ & $F_{I}$ \\
Loading state $(L)$ & $\sqrt{ }$ & $\sqrt{ }$ & $\sqrt{ }$ & $G_{L}$ & $F_{L}$ \\
\hline
\end{tabular}

Note: $\times=$ excluding the corresponding generalized loads; $\sqrt{ }=$ including the corresponding generalized loads.

Table 2. Three typical states and state parameters for a numerical model

\begin{tabular}{|c|c|c|c|c|c|c|}
\hline \multirow{2}{*}{$\begin{array}{l}\text { Typical } \\
\text { states }\end{array}$} & \multicolumn{3}{|c|}{ Loads considered } & \multirow{2}{*}{$\begin{array}{c}\text { Solving } \\
\text { state }\end{array}$} & \multicolumn{2}{|c|}{ State parameters } \\
\hline & Initial strain & self-weight & outer loads & & geometry & force \\
\hline Zero state $(Z)$ & $\sqrt{ }$ & $x$ & $x$ & Before solving & $G_{Z}$ & $F_{Z}$ \\
\hline Initial state $(I)$ & $\sqrt{ }$ & $\sqrt{ }$ & $x$ & Solution is done & $G_{I}$ & $F_{I}$ \\
\hline Loading state $(L)$ & $\sqrt{ }$ & $\sqrt{ }$ & $\sqrt{ }$ & Solution is done & $G_{L}$ & $F_{L}$ \\
\hline
\end{tabular}

There are six state parameters $\left(G_{Z}, F_{Z} ; G_{I}, F_{I} ; G_{L}, F_{L}\right)$ at three different states in Tables 1-2. To build a numerical model, $G_{Z}$ and $F_{Z}$ at the zero state are the two essential parameters. Thus, the determination of $G z$ and $F_{Z}$ is a key step for the following design and analysis. The determination of $G_{Z}$ or $F_{Z}$ is so-called form finding and force finding, respectively. Because of the rigid upper latticed shell, the discrepancy of geometric configurations between the zero state and the initial state is rather slight, and therefore, form finding analysis is usually a dispensable for the sake of simplification of the design (Cao and Zhang [11]). In this paper, the geometric configuration at the zero state $(G z)$ is taken as a known parameter that is equal to the geometric configuration from the architectural drawings. When the geometric configurations at the zero state $\left(G_{Z}\right)$ and the internal force at the initial state or loading state $\left(F_{I}\right.$ or $\left.F_{L}\right)$ are known, this paper will mainly study the force finding, which is the problem of determining the initial strain $F_{Z}$ at the zero state.

The pre-stress in the suspended-dome will appear only if the structure is tensioned according to a certain tension process; thus, the tension process should be considered during the force finding. During practical construction, we directly apply the force to the tension elements, which are so-called active tension elements, according to the given construction process. Other elements are so-called passive tension elements, which include the components of a single-layer latticed shell. For the numerical model, we apply the internal force to the active elements according to the given construction process at the zero state. In other words, the construction process will determine the components on which the internal force will be applied at the zero state. Thus, the upper force-finding problem can be described as follows.

$$
\left\{\begin{array}{l}
F_{I}\left(o r F_{L}\right)=\Psi\left(F_{Z}\right) \\
\text { s.t. special construction process }
\end{array}\right.
$$

Because the upper function $\Psi$ cannot be described in terms of a mathematical concept, force finding of a suspended-dome is in a problem category that cannot be formulated as an algorithm. Thus, $F_{Z}$ cannot be calculated by $\Psi^{-1}$. In reference (Guo et al [13]), $F_{Z}$ was solved by Newton iteration. However, the associated developed methods are inconvenient for engineers to operate.

From Eq. 1, we can see that we input any $F_{Z}$ that conforms to a special construction process; then, the corresponding $F_{I}$ (or $F_{L}$ ) can be output. This paper will use the BP network to address the upper fuzzy function between $F_{Z}$ and $F_{I}\left(\right.$ or $\left.F_{L}\right)$. 


\section{BP NEURAL NETWORKS}

Artificial neural networks (ANN) consist of very simple but numerous nerve cells that work massively in parallel and that have the capability to learn. The main characteristics of ANNs are self-organization learning capability, generalization capability and fault tolerance. An ANN has an excellent ability to learn and to describe the highly nonlinear and strongly coupled relationships between multiple-input and multiple-output variables (Kriesel [17]). The basic structure of the neuron is shown in Figure 1.

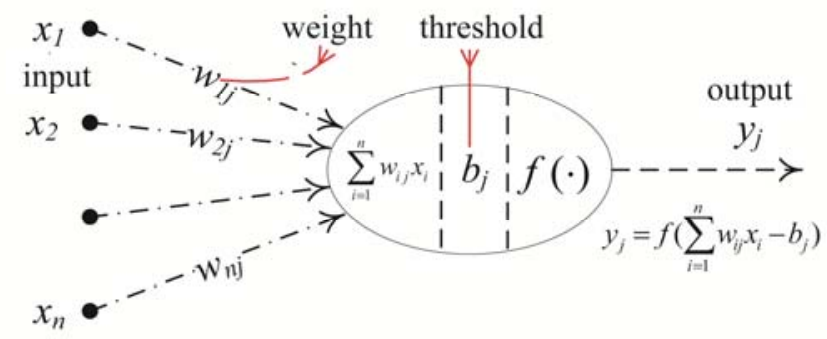

Figure 1. The Basic Structure of the Neuron

where $x_{n}$ is the $n$th input signal, $w_{n j}$ is the connection weight from the $n$th input neuron to the $j$ th neuron, $b_{j}$ is the threshold value of the $j$ th neuron, and $y_{j}$ is the output signal of the $j$ th neuron. The neuron output signal $y_{j}$ is calculated by the activation function, as follows:

$y_{j}=f\left(\sum_{i=1}^{n}\left(w_{i j} x_{i}\right)-b_{j}\right)$

Many types of activation functions have been proposed, among which the most popular activation function for back-propagation networks (BP) is the sigmoid, which is expressed as follows:

$f(x)=\frac{1}{1+e^{-c x}}$

In this study, the value of the parameter $c$ is 1 .

The BP neural network is a multilayer feed forward network, whose basic network structure for the upper force finding can be built as shown in Figure 2.

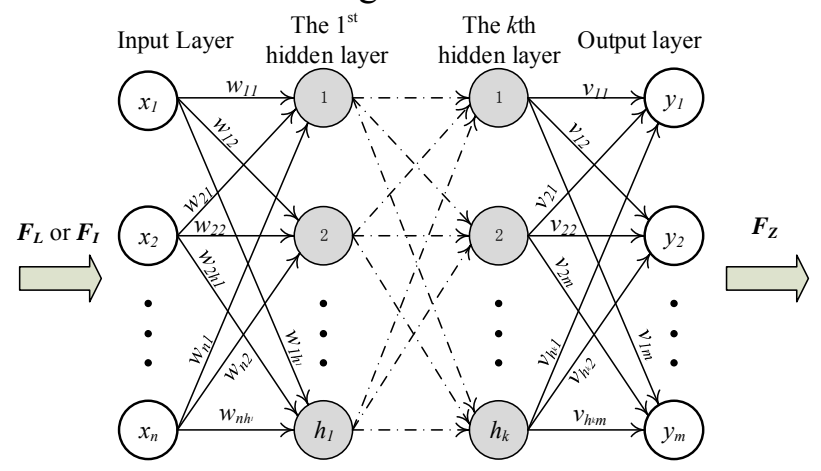

Figure 2. The Structure of the BP Network 
The network with $n$ input and $m$ output neurons is clearly delineated: there is one input layer, one output layer and one or more hidden layers. Each layer consists of several neurons, and the layers are interconnected by sets of correlation weights. In the BP neural network, the error at the output layer propagates backwards to the input layer through one or more hidden layers to obtain the desired outputs.

The processes of the BP network for solving the force finding include the following three steps:

Step 1: Establishing the network

The number of the input and output neurons $(n, m)$ and the hidden layer $(k)$ is first determined according to a practical tension process.

Then, the number of units in $i$ th hidden layer $\left(h_{i}\right)$ is determined according to the number of the input and output neurons. Gao [18] studied many practical examples on calculating the number of units in the hidden layer and gave a fitting empirical formula by the least square method, as follows. The empirical formula will be used in this paper.

$h_{i}=\sqrt{0.43 m n+0.12 m^{2}+2.54 n+0.77 m+0.35}+0.51$

Last, the connection weights $\left(w_{i j}\right)$ and the thresholds $\left(b_{j}\right)$ are initialized to values between -1 and +1 , randomly.

Step 2: Training the network

Training the network is the process of repeatedly modifying the connection weights $\left(w_{i j}\right)$ and the thresholds $\left(b_{j}\right)$ according to the difference between the output patterns and the desired output patterns until the optimal weights and thresholds appear. The detailed process of training the network is the following: First, we can obtain a training set that consists of input patterns $[X]_{a \times n}$ and the corresponding output patterns $[Y]_{a \times m}$ by experiments or numerical analysis. Then, we apply the inputs to the network and compute the prediction outputs $[P]_{a \times m}$. We compare $[P]_{a \times m}$ with $[Y]_{a \times m}$ and provide the error vector. Last, we change the weights $\left(w_{i j}\right)$ and the thresholds $\left(b_{j}\right)$ based on the error vector. Generally, the training will stop when the error is small enough.

Step 3: Generalization (or Prediction)

The most interesting characteristic of neural networks is their capability to familiarize with problems by means of training and, after sufficient training, to be able to solve unknown problems of the same class. Thus, we can utilize the trained network to generalize the results of force finding, corresponding to the known values $\tilde{N}$. If the prediction errors do not meet the allowable value $\varepsilon$, then we should expend the scale of the training patterns and then repeat the above steps, namely, step 2 and step 3 .

The initial weights and thresholds will influence the training difficulty and generalization error because they are random numbers mentioned in step 1. Thus, this paper will use a Genetic Algorithm (GA) to optimize the initial weights and thresholds of the BP network.

The optimization starts from the first population that consists of many randomly generated individuals, which include the weights and the thresholds of the BP network arranged in linear succession. Then, the BP network with different individuals is trained by input data $[X]_{a \times n}$ and output data $[Y]_{a \times m}$, and the absolute error between the output $[Y]_{a \times m}$ and the prediction output 
$[P]_{a \times m}$ for different individuals is calculated and set as the individual fitness. The fitness of the optimal individual is minimal among all of the individuals. Subsequently, a Roulette Wheel Selection generates a new population. Some of the individuals undergo transformations by means of crossover and mutation. This process occurs when moving from the current population to the next population and constitutes one generation in the genetic algorithm evolutionary process. After some generations, the program converges to a feasible solution in which the best individual represents a near-to-optimum solution.

The BP network and the GA were programmed and executed by using the neural network toolbox and GA toolbox of the MATLAB (version 7.14, 2012a) software. All of the input and output data were generated by ANSYS software. The whole process described above is summarized in the flowchart of Figure 3.

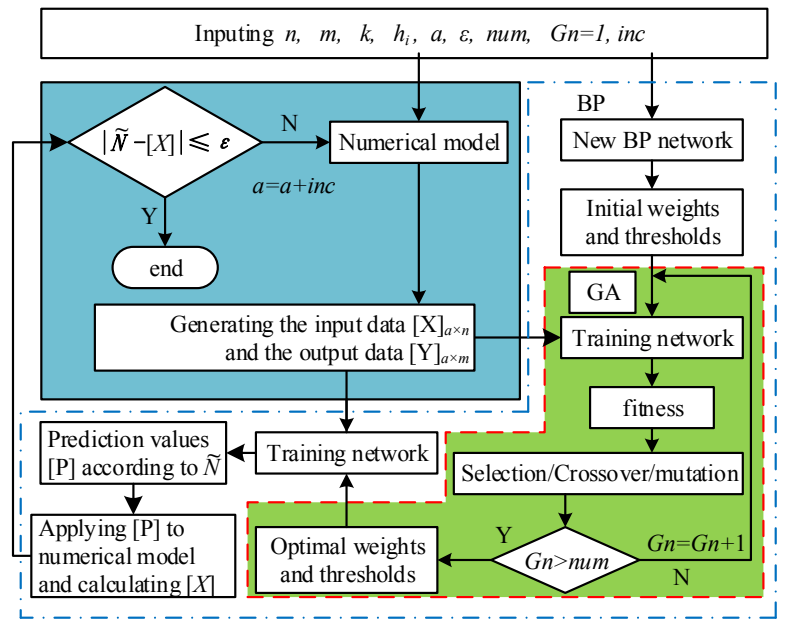

Figure 3. Flowchart of Force Finding for the Suspended-dome with BP

\section{AN ILLUSTRATIVE EXAMPLE}

The numerical model shown in Figure 4 is selected as an illustrative example, which considers a span and rise of $122 \mathrm{~m}$ and $12.2 \mathrm{~m}$, respectively. Its lower cable-strut arrangement is a rib pattern that includes three rings of cables and struts. In addition, the concrete lattice pattern of the upper single-layer latticed shell is a hybrid pattern that combines the Keiwitt pattern with a sunflower pattern. Table 3 presents the cross-sectional area and the material properties of radial cables, hoop cables, struts and all components in the single-layer latticed shell. This paper assumes that the internal forces of the hoop cables at an initial state from the outermost to the innermost ring are $2500 \mathrm{kN}(\mathrm{H} 1), 1000 \mathrm{kN}(\mathrm{H} 2)$, and $500 \mathrm{kN}(\mathrm{H} 3)$, respectively. In other words, the $F_{I}$ of the suspended-dome is a known value that can be expressed as $\tilde{N}=[2500 \mathrm{kN}, 1000 \mathrm{kN}, 500 \mathrm{kN}]$, and the geometry configuration at the zero state $\left(G_{Z}\right)$ is described in Figure 4 . The numerical model will be analyzed by the ANSYS program. The element types for the components in the numerical model are also listed in Table 3. In the following research, the suspended-dome will be constructed by three different tension processes: tensioning hoop cables, tensioning radial cables and jacking struts. 
Table 3. Geometrical and Material Properties for the Components of the Numerical Model

\begin{tabular}{lllll}
\hline Members & Sectional area $/ \mathrm{m}^{2}$ & density $/ \mathrm{kg} / \mathrm{m}^{3}$ & Young's modulus E/ pa & Element type \\
\hline $\mathrm{H} 1$ & 0.01124 & $6.55 \times 10^{3}$ & $1.9 \times 10^{11}$ & Link10 \\
$\mathrm{H} 2$ & 0.00562 & $6.55 \times 10^{3}$ & $1.9 \times 10^{11}$ & Link10 \\
$\mathrm{H} 3$ & 0.00285 & $6.55 \times 10^{3}$ & $1.9 \times 10^{11}$ & Link10 \\
$\mathrm{R} 1$ & 0.00562 & $6.55 \times 10^{3}$ & $1.9 \times 10^{11}$ & Link10 \\
$\mathrm{R} 2$ & 0.00285 & $6.55 \times 10^{3}$ & $1.9 \times 10^{11}$ & Link10 \\
$\mathrm{R} 3$ & 0.00285 & $6.55 \times 10^{3}$ & $1.9 \times 10^{11}$ & Link10 \\
S1 & 0.004662 & $7.85 \times 10^{3}$ & $2.06 \times 10^{11}$ & Link8 \\
S2 & 0.004662 & $7.85 \times 10^{3}$ & $2.06 \times 10^{11}$ & Link8 \\
S3 & 0.004662 & $7.85 \times 10^{3}$ & $2.06 \times 10^{11}$ & Link8 \\
Single layer & $\varnothing 377 \times 12$ & $7.85 \times 10^{3}$ & $2.06 \times 10^{11}$ & Beam188 \\
latticed shell & & & & \\
\hline
\end{tabular}

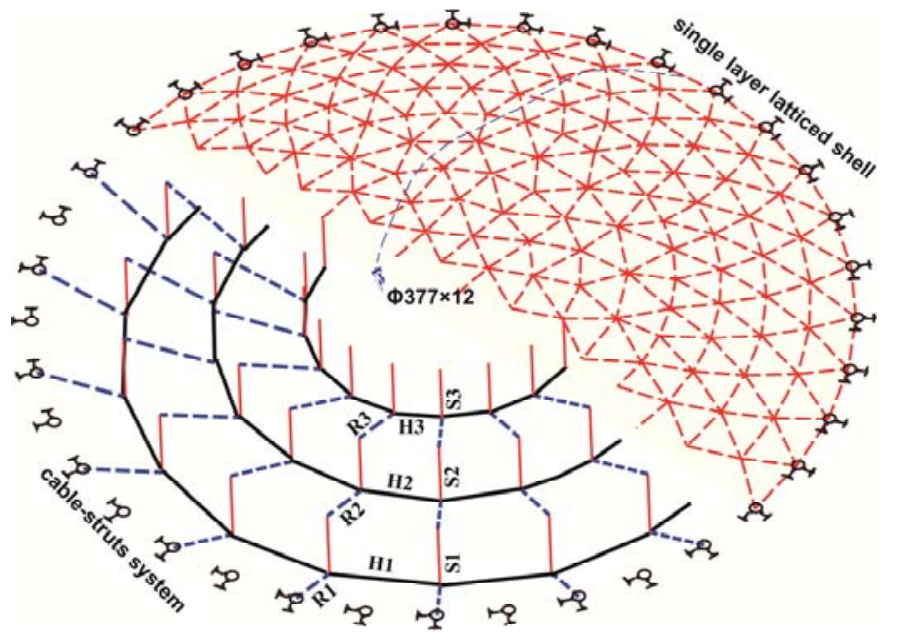

(a) Numerical model (perspective view), ( $\mathrm{S}=\mathrm{Struts}, \mathrm{H}=$ Hoop cables and $\mathrm{R}=$ Radial cables)

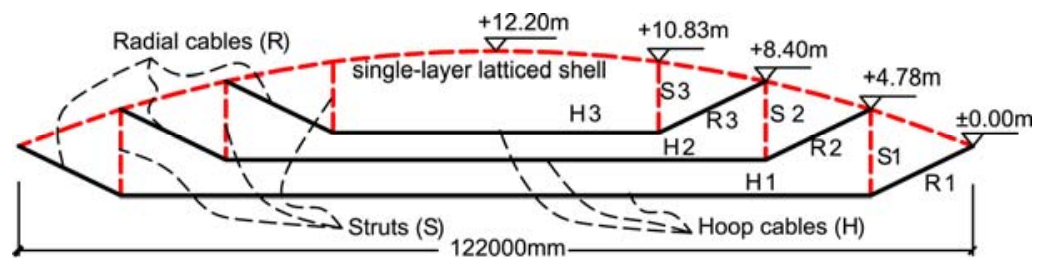

(b) Numerical model (vertical view)

Figure 4. Numerical Model of Suspended-dome

During the force finding, the internal force at the zero state will be simulated by the initial strains, which are applied only to the corresponding active tension elements; these elements are hoop cables, radial cables and struts for tensioning the hoop cables, radial cables and jacking struts.

According to the known parameters and the construction process, the initial strain at the zero state and the internal force at the initial state are selected as the input data and the output data for the BP network, respectively. The detailed sources of the input data and the output data under different tension processes are listed in Table 4. 
Table 4. Input and Output Data under Different Construction Processes

\begin{tabular}{lllllll}
\hline \multirow{2}{*}{ Construction process } & \multicolumn{2}{l}{ Input data } & \multicolumn{3}{l}{ Output data } \\
\cline { 2 - 6 } & \multicolumn{2}{l}{$F_{I}$ (internal forces) } & \multicolumn{3}{l}{ (initial strains) } \\
\hline Tensioning hoop cables & H1 & H2 & H3 & H1 & H2 & H3 \\
Tensioning radial cables & H1 & H2 & H3 & R1 & R2 & R3 \\
Jacking struts & H1 & H2 & H3 & S1 & S2 & S3 \\
\hline
\end{tabular}

From Table 4, we can symbolize the input data and the corresponding output data as follows:

$$
\begin{aligned}
& {[X]_{a \times 3}=\left[\begin{array}{lll}
F_{I}^{1}, & F_{I}^{2}, & F_{I}^{3}
\end{array}\right]_{a}} \\
& {[Y]_{a \times 3}=\left[\begin{array}{lll}
F_{z}^{1}, & F_{z}^{2}, & F_{z}^{3}
\end{array}\right]_{a}}
\end{aligned}
$$

where $a$ is the data size.

A simple three-layered neural network with an input layer, a hidden layer and an output layer is adopted in this paper. According to Eq. 4, the number of units in the hidden layer is set to 5 . Thus, the structure of the BP network for force finding is $3 \times 5 \times 3$.

Generally, the training data (the input and the output) for the BP network can be randomly generated according to the given tension process. At the same time, to allow the training data, especially the input data, approach the known values of $\tilde{N}$, this paper restricts the range of the training data by the following process:

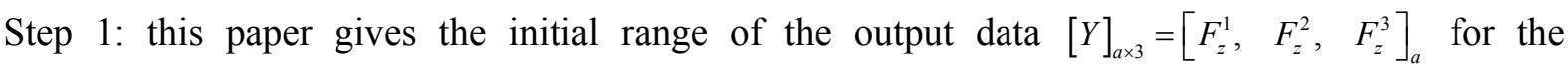
corresponding active elements, as follows:

$$
\begin{aligned}
& \beta \frac{1 \times 10^{4}}{E A_{i}} \leq F_{z}^{i} \leq \gamma \frac{1 \times 10^{4}}{E A_{i}} \quad(i=1,2,3) \\
& \left|E A_{1} F_{z}^{1}\right|>\left|E A_{2} F_{z}^{2}\right|>\left|E A_{2} F_{z}^{3}\right|
\end{aligned}
$$

where $E$ and $A_{1}, A_{2}, A_{3}$ are the young's modules and sectional area of the corresponding active elements, and $\beta$ and $\gamma$ are the random values. Here, this paper allows $\beta=1, \gamma=20$ for tensioning the hoop cables and the radial cables and $\beta=-20, \gamma=-1$ for the jacking struts.

Step 2: this paper generates training data in the upper range and obtains the prediction values ${ }^{p} F_{z}^{i}$ that correspond to the known values $\tilde{N}$.

Step 3: the new range for the training data is given as follows:

$$
\begin{aligned}
& \eta_{1}{ }^{p} F_{z}^{i} \leq F_{z}^{i} \leq \eta_{2}{ }^{p} F_{z}^{i} \quad(i=1,2,3) \\
& \left|E A_{1} F_{z}^{1}\right|>\left|E A_{2} F_{z}{ }^{2}\right|>\left|E A_{3} F_{z}^{3}\right|
\end{aligned}
$$

where $\eta_{1}$ and $\eta_{2}$ are random values. Here, this paper allows $\eta_{1}=0.9$ and $\eta_{2}=1.1$ for tensioning the hoop cables and the radial cables and $\eta_{1}=1.1, \eta_{2}=0.9$ for the jacking struts.

According to the upper process, this paper first generated 100 patterns randomly according to step 1 , some of which were listed in Table 5. 
Then, this paper applied the training data to the network without optimizing by the GA and predicted the output data that corresponds to the known input data $\tilde{N}$ five times, according to the scheme in Figure 3. During the prediction, the BP network will be trained by the Levenberg-Marquardt method, which is a perfect approach for small-size networks. Additionally, the technical parameters of the BP network are set as: net.trainParam. $1 \mathrm{r}=0.01$ and net.trainParam.goal $=0.0000001$. The active function in the hidden layer will adopt the tan-sigmoid transform function ( $\operatorname{tansig}())$, and the output layer is the linear function (purelin()). The prediction results that correspond to the three different construction processes at the zero state are summarized in Table 6.

Table 5. Partial Training Samples for Three Different Construction Processes

\begin{tabular}{|c|c|c|c|c|c|c|c|}
\hline \multirow{2}{*}{$\begin{array}{l}\text { construction } \\
\text { process }\end{array}$} & \multirow{2}{*}{ NO } & \multicolumn{3}{|c|}{ input data $/ \mathrm{kN}$} & \multicolumn{3}{|c|}{ output data $/\left(\times 10^{4} / \mathrm{EA}_{\mathrm{j}}\right)$} \\
\hline & & $\mathrm{H} 1$ & $\mathrm{H} 2$ & $\mathrm{H} 3$ & $\mathrm{H} 1$ & $\mathrm{H} 2$ & $\mathrm{H} 3$ \\
\hline \multirow{5}{*}{ Hoop } & 1 & 13710.230 & 5978.154 & 3340.706 & 1709.632 & 968.652 & 837.188 \\
\hline & 2 & 12515.590 & 6849.735 & 4264.299 & 1415.498 & 1274.050 & 1121.364 \\
\hline & $\ldots$ & $\ldots$ & $\ldots$ & $\ldots$ & $\ldots$ & $\ldots$ & $\ldots$ \\
\hline & 99 & 8778.814 & 3883.778 & 1332.480 & 1091.725 & 697.483 & 241.485 \\
\hline & 100 & 15291.340 & 7851.650 & 2534.924 & 1830.718 & 1519.926 & 442.369 \\
\hline \multirow{6}{*}{ Radial } & & $\mathrm{H} 1$ & $\mathrm{H} 2$ & $\mathrm{H} 3$ & $\mathrm{R} 1$ & $\mathrm{R} 2$ & R3 \\
\hline & 1 & 9452.421 & 4886.658 & 2713.418 & 1709.632 & 968.652 & 837.188 \\
\hline & 2 & 8927.791 & 5775.201 & 3503.986 & 1415.498 & 1274.050 & 1121.364 \\
\hline & $\ldots$ & $\ldots$ & $\ldots$ & $\ldots$ & $\ldots$ & $\ldots$ & ... \\
\hline & 99 & 6091.049 & 3188.418 & 1079.992 & 1091.725 & 697.483 & 241.485 \\
\hline & 100 & 10741.470 & 6587.421 & 2079.908 & 1830.718 & 1519.926 & 442.369 \\
\hline \multirow{6}{*}{ Struts } & & $\mathrm{H} 1$ & $\mathrm{H} 2$ & $\mathrm{H} 3$ & S1 & $\mathrm{S} 2$ & S3 \\
\hline & 1 & 2020.409 & 831.240 & 281.247 & -1207.785 & -941.683 & -379.918 \\
\hline & 2 & 2946.826 & 1206.958 & 379.722 & -1817.063 & -1354.235 & -429.386 \\
\hline & $\ldots$ & $\ldots$ & $\ldots$ & $\ldots$ & $\ldots$ & $\ldots$ & $\ldots$ \\
\hline & 99 & 2432.193 & 976.260 & 486.024 & -1478.619 & -978.630 & -911.769 \\
\hline & 100 & 3051.062 & 1401.979 & 688.145 & -1773.837 & -1626.241 & -1268.992 \\
\hline
\end{tabular}

Table 6. The prediction value under different construction processes

\begin{tabular}{|c|c|c|c|c|c|c|c|c|c|}
\hline \multirow{2}{*}{$\begin{array}{l}\text { Prediction } \\
\text { times }\end{array}$} & \multicolumn{3}{|c|}{$\begin{array}{c}\text { Tension hoop } \\
\text { cable } /\left(\times 10^{4} / \mathrm{EA}_{\mathrm{j}}\right)\end{array}$} & \multicolumn{3}{|c|}{$\begin{array}{c}\text { Tension radial } \\
\text { cable } /\left(\times 10^{4} / \mathrm{EA}_{\mathrm{j}}\right)\end{array}$} & \multicolumn{3}{|c|}{ Jack struts $/\left(\times 10^{4} / \mathrm{EA}_{\mathrm{j}}\right)$} \\
\hline & $\mathrm{H} 1$ & $\mathrm{H} 2$ & $\mathrm{H} 3$ & $\mathrm{R} 1$ & $\mathrm{R} 2$ & R3 & S1 & S2 & S3 \\
\hline 1 & 304.78 & 160.90 & 132.53 & 474.26 & 170.61 & 156.38 & -1524.88 & -995.28 & -937.98 \\
\hline 2 & 307.00 & 163.35 & 135.33 & 474.09 & 171.69 & 156.57 & -1524.51 & -995.61 & -938.10 \\
\hline 3 & 347.04 & 218.33 & 156.33 & 473.30 & 165.53 & 163.50 & -1524.46 & -996.00 & -936.93 \\
\hline 4 & 299.93 & 165.92 & 132.39 & 473.69 & 169.49 & 157.095 & -1524.72 & -995.45 & -938.07 \\
\hline 5 & 300.24 & 143.12 & 134.26 & 471.85 & 176.55 & 149.67 & -1525.38 & -995.60 & -937.26 \\
\hline
\end{tabular}

Table 6 shows that the results from the different prediction times are different. To find out the accuracy of the prediction values, this paper applied the prediction values $[P]_{5 \times 3}$ in Table 6 to the numerical model and output the corresponding internal force of the hoop cable at the initial state $\left[{ }^{p} F_{I}^{1},{ }^{p} F_{I}^{2},{ }^{p} F_{I}^{3}\right]_{5 \times 3}$. The relative errors between the prediction value $\left[{ }^{p} F_{I}^{1},{ }^{p} F_{I}^{2}, \quad{ }^{p} F_{I}^{3}\right]_{5 \times 3}$ and the known value $\tilde{N}$ under three different construction processes are obtained by the following Eq. 9 . And, the results of the relative errors are shown in Table 7.

Error $=(P-\tilde{N}) / \tilde{N} \mid \times 100$ 
Table 7. The Prediction Errors of Three Different Construction Processes for Different Times

\begin{tabular}{llllllllll}
\hline \multirow{2}{*}{$\begin{array}{l}\text { Prediction } \\
\text { times }\end{array}$} & \multicolumn{2}{c}{ Tension hoop cable/(\%) } & \multicolumn{3}{c}{ Tension radial cable/(\%) } & \multicolumn{3}{c}{ Jack struts/(\%) } \\
\cline { 2 - 10 } & H1 & H2 & H3 & H1 & H2 & H3 & H1 & H2 & H3 \\
\hline 1 & 0.9604 & 1.9414 & 0.1525 & 0.4593 & 1.4835 & 0.3039 & 0.0009 & 0.0065 & 0.0026 \\
2 & 1.8016 & 3.1890 & 1.6394 & 0.5156 & 1.7977 & 0.1188 & 0.0140 & 0.0007 & 0.0057 \\
3 & 17.0820 & 27.2459 & 20.7601 & 0.0739 & 0.3013 & 2.0867 & 0.0161 & 0.0052 & 0.0692 \\
4 & 0.0839 & 2.8397 & 0.0245 & 0.2980 & 1.1473 & 0.1452 & 0.0053 & 0.0025 & 0.0040 \\
5 & 1.5764 & 4.1127 & 1.5597 & 0.3570 & 2.6503 & 2.5837 & -0.0281 & -0.0161 & 0.0388 \\
\hline
\end{tabular}

Table 7 shows that the prediction errors are different after each prediction. Moreover, the partial prediction errors are large, such as $27.2459 \%, 20.7601 \%$ and $17.0820 \%$. Thus, this paper selected the prediction values that correspond to the minimal prediction errors for the new range. From Table 7 , we can see that the minimal prediction errors for tensioning hoop cables, radial cables and jacking struts appear in the $1^{\text {st }}$ prediction, $4^{\text {th }}$ prediction and $5^{\text {th }}$ prediction, respectively. Additionally, the corresponding prediction values in Table 6 will be applied to Eq. 8 to obtain the new range for the training data. According to the new range, this paper generates 1000 patterns for three different construction processes randomly. Partial patterns from different construction processes are listed in Table 8.

Table 8. Partial Training Samples under Tensioning Radial Cable

\begin{tabular}{lccccccc}
\hline Construction & \multirow{2}{*}{$\begin{array}{c}\text { NO } \\
\text { process }\end{array}$} & \multicolumn{3}{c}{ Input data $/ \mathrm{kN}$} & \multicolumn{3}{c}{ Output data/ $\left(\times 10^{4} / \mathrm{EA}_{\mathrm{j}}\right)$} \\
\cline { 3 - 8 } & 1 & 2680.105 & 1079.136 & 529.696 & 325.409 & 169.461 & 140.069 \\
\multirow{4}{*}{ Hoop } & 2 & 2501.329 & 1047.818 & 479.478 & 298.013 & 175.046 & 122.271 \\
& $\ldots$ & $\ldots$ & $\ldots$ & $\ldots$ & $\ldots$ & $\ldots$ & $\ldots$ \\
& 999 & 2324.651 & 965.419 & 507.561 & 275.263 & 154.616 & 141.659 \\
& 1000 & 2376.157 & 969.901 & 477.306 & 284.363 & 154.926 & 127.860 \\
\hline \multirow{4}{*}{ Radial } & & $\mathrm{H} 1$ & $\mathrm{H} 2$ & $\mathrm{H} 3$ & $\mathrm{R} 1$ & $\mathrm{R} 2$ & $\mathrm{R} 3$ \\
\cline { 3 - 8 } & 2414.133 & 987.197 & 497.898 & 452.023 & 167.222 & 159.386 \\
& 2 & 2396.334 & 964.696 & 464.533 & 451.746 & 162.410 & 144.450 \\
& $\ldots$ & $\ldots$ & $\ldots$ & $\ldots$ & $\ldots$ & $\ldots$ & $\ldots$ \\
& 999 & 2410.120 & 999.108 & 491.172 & 449.055 & 172.979 & 155.047 \\
& 1000 & 2395.793 & 965.541 & 457.816 & 451.619 & 163.387 & 140.965 \\
\hline \multirow{4}{*}{ Struts } & & $\mathrm{H} 1$ & $\mathrm{H} 2$ & $\mathrm{H} 3$ & $\mathrm{~S} 1$ & $\mathrm{~S} 2$ & $\mathrm{~S} 3$ \\
& 1 & 2444.214 & 1021.781 & 482.025 & -1463.575 & -1091.760 & -875.070 \\
& 2 & 2479.843 & 999.123 & 481.570 & -1508.682 & -1014.435 & -883.092 \\
& $\ldots$ & $\ldots$ & $\ldots$ & $\ldots$ & $\ldots$ & $\ldots$ & $\ldots$ \\
& 999 & 2573.127 & 988.632 & 469.207 & -1599.524 & -944.116 & -844.347 \\
& 1000 & 2672.678 & 1012.820 & 488.722 & -1673.499 & -941.794 & -885.315 \\
\hline
\end{tabular}

To know the influence of the sample size on the prediction accuracy of the BP network, this paper selected the training data from 1000 patterns in sequence and created 19 groups of training data whose sizes are $10,20,30,40,50,60,70,80,90,100,200,300,400,500,600,700,800,900$, and 1000. Then, this paper applied these 19 groups of training data to the network without optimizing with the GA and predicted the output data that corresponds to the known input data $\tilde{N}$ according to the scheme in Figure 3. The partial prediction results, which are found from the samples of different sizes, correspond to three different construction processes at the zero state and are summarized in Table 9. 
Table 9. The Prediction Values under Different Construction Processes

\begin{tabular}{|c|c|c|c|c|c|c|c|c|c|}
\hline \multirow{2}{*}{$\begin{array}{l}\text { sample } \\
\text { size }\end{array}$} & \multicolumn{3}{|c|}{$\begin{array}{c}\text { Tension hoop } \\
\text { cable } /\left(\times 10^{4} / \mathrm{EA}_{\mathrm{j}}\right)\end{array}$} & \multicolumn{3}{|c|}{$\begin{array}{c}\text { Tension radial } \\
\text { cable } /\left(\times 10^{4} / \mathrm{EA}_{\mathrm{j}}\right)\end{array}$} & \multicolumn{3}{|c|}{ Jack struts $/\left(\times 10^{4} / \mathrm{EA}_{\mathrm{j}}\right)$} \\
\hline & H1 & $\mathrm{H} 2$ & $\mathrm{H} 3$ & R1 & R2 & R3 & S1 & S2 & S3 \\
\hline 10 & 276.606 & 149.411 & 148.956 & 458.904 & 163.600 & 156.247 & -1538.206 & 1045.117 & -986.046 \\
\hline 20 & 302.812 & 155.153 & 134.301 & 473.350 & 165.249 & 158.512 & -1524.708 & -995.024 & -937.805 \\
\hline$\cdots$ & $\cdots$ & $\cdots$ & $\cdots$ & $\cdots$ & & & $\cdots$ & $\cdots$ & $\cdots$ \\
\hline 90 & 302.632 & 155.236 & 134.411 & 473.556 & 165.361 & 158.476 & -1524.820 & -995.431 & -937.949 \\
\hline$\ldots$ & $\ldots$ & $\ldots$ & $\ldots$ & $\cdots$ & $\cdots$ & $\ldots$ & & $\ldots$ & \\
\hline 500 & 302.629 & 155.235 & 134.415 & 473.575 & 165.365 & 158.479 & & -995.443 & -937.997 \\
\hline$\cdots$ & $\cdots$ & & $\cdots$ & $\cdots$ & & $\cdots$ & & & \\
\hline 900 & 302.637 & 155.229 & 134.420 & 473.561 & 165.364 & 158.474 & -1524.711 & -995.430 & -938.017 \\
\hline 1000 & 302.627 & 155.237 & 134.412 & 473.558 & 165.359 & 158.477 & -1524.810 & -995.424 & -937.989 \\
\hline
\end{tabular}

Similar to the above process, this paper applied the prediction values $[P]_{19 \times 3}$ in Table 9 to the numerical model and output the corresponding internal force of the hoop cable at the initial state $\left[{ }^{p} F_{I}^{1},{ }^{p} F_{I}^{2},{ }^{p} F_{I}^{3}\right]_{19 \times 3}$. The results of the relative errors are shown in Figure 5.

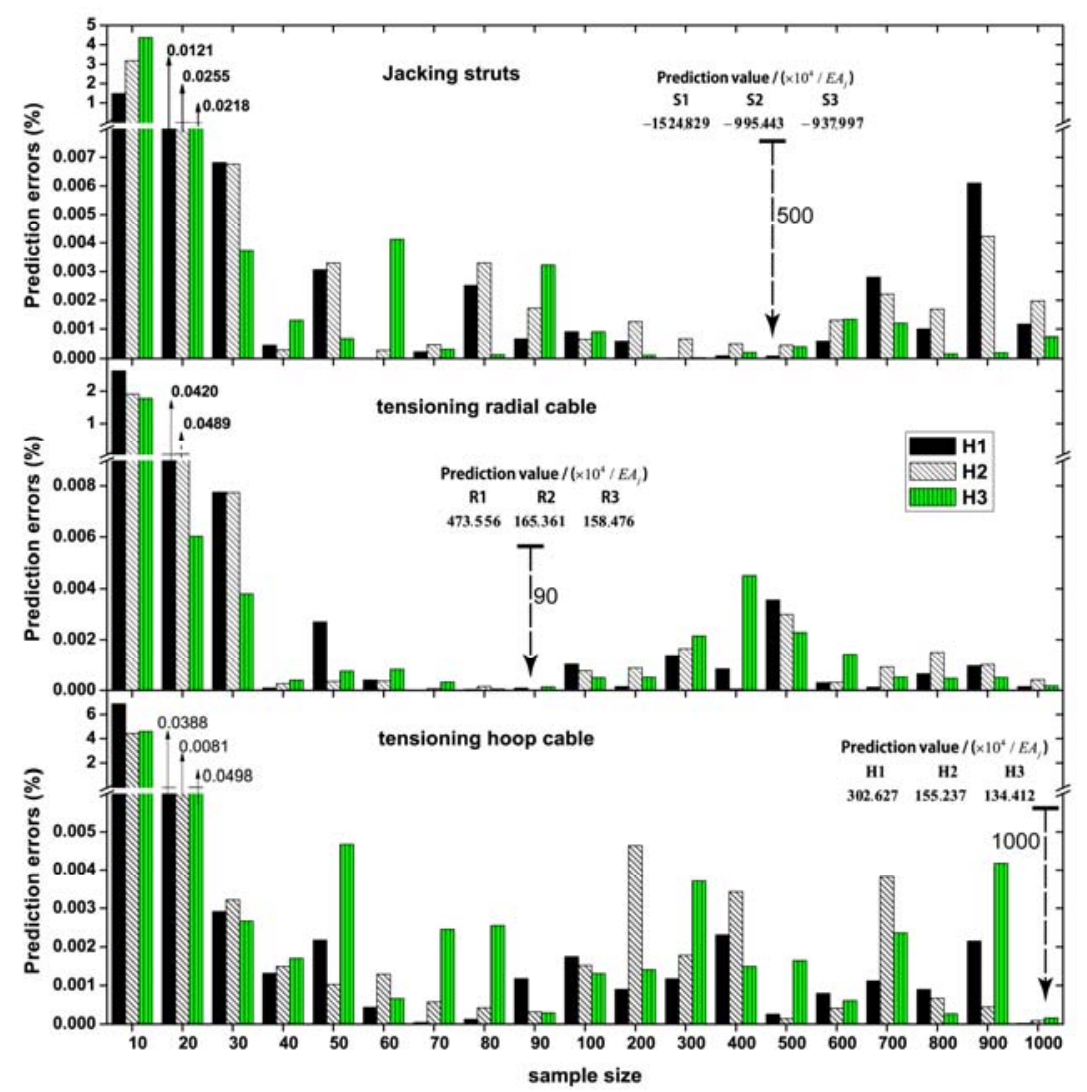

Figure 5. Distribution of the Prediction Errors from Different Construction Processes

The prediction errors in Figure 5 are significantly less than those in Table 7. Thus, the method for determining the range for the training data according to the previous prediction values is helpful for enhancing the prediction precision. At the same time, Figure 5 shows that the increase in the training sample size can generally enhance the prediction precision. The prediction errors are significantly reduced with the increase in the training sample size when the sample size is less than 40 . However, after the sample size reaches a certain value, such as 50 , the influence of the sample size on the prediction errors will decrease. When the sample size is equal to or greater than 50 , the prediction errors of the tensioning hoop cables, tensioning radial cables and jacking struts are less than $0.005 \%$, 
$0.005 \%$, and $0.006 \%$, respectively. In general, the BP network can accurately predict the results of the force finding for the suspended-dome. At the same time, from Figure 5, we can see that the prediction errors are minimal when the sample size is 1000,90 , and 500 for the tensioning hoop cables, radial cables and jacking struts. The corresponding results of the force finding are listed in Table 9. The sample size that corresponds to the minimal prediction error is not always the maximal size of 1000 . At the same time, Table 6 and Table 7 show that the same network and same training data gained different prediction results after each prediction, and the prediction errors vary from $0.0245 \%$ to $27.2459 \%$. The main cause of this result is that the random numbers initialized the connection weights and thresholds for the training.

To evaluate the influence of the initial weights and thresholds on the prediction, in this study, we selected 1000 sample data on tensioning the radial cables and used the genetic algorithm (GA) mentioned in section 4 to optimize the 30 weights and eight thresholds of the BP. Then, we trained and predicted the values that correspond to the known values 10 times, according to the scheme in Figure 3.

During the optimization, we took the sum of the errors between the output data and the corresponding prediction values as the fitness of the optimization, which can be expressed as follows:

$$
f(i)=\sum_{l=1}^{s} \sum_{j=1}^{3}\left(\left|{ }^{\mathrm{i}} F_{z}^{j l}-{ }_{p}^{i} F_{z}^{j l}\right|\right)
$$

Each run of the GA is conducted for $40(i=1,2, \cdots 40)$ generations while using a population size of $80(s=80)$. Proportional selection is employed to probabilistically select genotypes for crossover and mutation. Two pairs for a pairwise single-point crossover operation and uniform mutation operations were used. The crossover rate and the mutation rate were empirically set to 0.3 and 0.2 .

We also applied 1000 sample data to the network without optimizing by the GA to predict the output data that corresponds to the known input data 10 times. The goal in this case was to compare the prediction results between the BP optimized by GA and the BP without optimization. The prediction values from only the BP network and the BP network optimized by the GA are summarized in Table 10.

Table 10. The Prediction Value for Tensioning Radial Cables by BP and BP $+\mathrm{GA}$

\begin{tabular}{|c|c|c|c|c|c|c|}
\hline \multirow{3}{*}{ times } & \multicolumn{6}{|c|}{ Initial strain at the zero state $/\left(\times 10^{4} / \mathrm{EA}_{\mathrm{j}}\right)$} \\
\hline & \multicolumn{3}{|c|}{ BP } & \multicolumn{3}{|c|}{$\mathrm{BP}+\mathrm{GA}$} \\
\hline & $\mathrm{R} 1$ & $\mathrm{R} 2$ & R3 & R1 & R2 & R3 \\
\hline 1 & 473.5541 & 165.3572 & 158.4735 & 473.5555 & 165.3614 & 158.4769 \\
\hline 2 & 473.5494 & 165.3471 & 158.4798 & 473.5536 & 165.3606 & 158.4799 \\
\hline 3 & 473.5671 & 165.3658 & 158.4801 & 473.5561 & 165.3612 & 158.4764 \\
\hline 4 & 473.5581 & 165.3546 & 158.4758 & 473.5553 & 165.3614 & 158.4769 \\
\hline 5 & 473.5534 & 165.3614 & 158.4744 & 473.5537 & 165.3604 & 158.4801 \\
\hline 6 & 473.5567 & 165.3695 & 158.4839 & 473.5561 & 165.3612 & 158.4765 \\
\hline 7 & 473.5598 & 165.3539 & 158.4807 & 473.5556 & 165.3613 & 158.4769 \\
\hline 8 & 473.5498 & 165.3392 & 158.4725 & 473.5560 & 165.3612 & 158.4764 \\
\hline 9 & 473.5593 & 165.3605 & 158.4756 & 473.5560 & 165.3612 & 158.4764 \\
\hline 10 & 473.5502 & 165.3603 & 158.4795 & 473.5516 & 165.3602 & 158.4804 \\
\hline $\operatorname{Mean}$ value $(\mu)$ & 473.5558 & 165.3570 & 158.4776 & 473.5549 & 165.3610 & 158.4777 \\
\hline $\begin{array}{l}\text { standard } \\
\text { deviation }(\boldsymbol{\sigma})\end{array}$ & 0.0056 & 0.0089 & 0.0037 & 0.0015 & 0.0004 & 0.0017 \\
\hline
\end{tabular}


Table 10 shows that the prediction values are different each time. In other words, the BP network with the same structure and the same training data has different prediction errors after different trainings. At the same time, we can see that the standard deviation $\sigma$ of $\mathrm{BP}+\mathrm{GA}$ is significantly less than that of BP. Thus, the prediction stability of the BP network will be improved after optimization by GA.

To know the prediction errors from $\mathrm{BP}$ and $\mathrm{BP}+\mathrm{GA}$, we applied the prediction values in Table 10 to the numerical model and obtained the internal force at the initial state; then, we calculated the relative errors by Eq. 9. The prediction errors are shown in Figure 6.

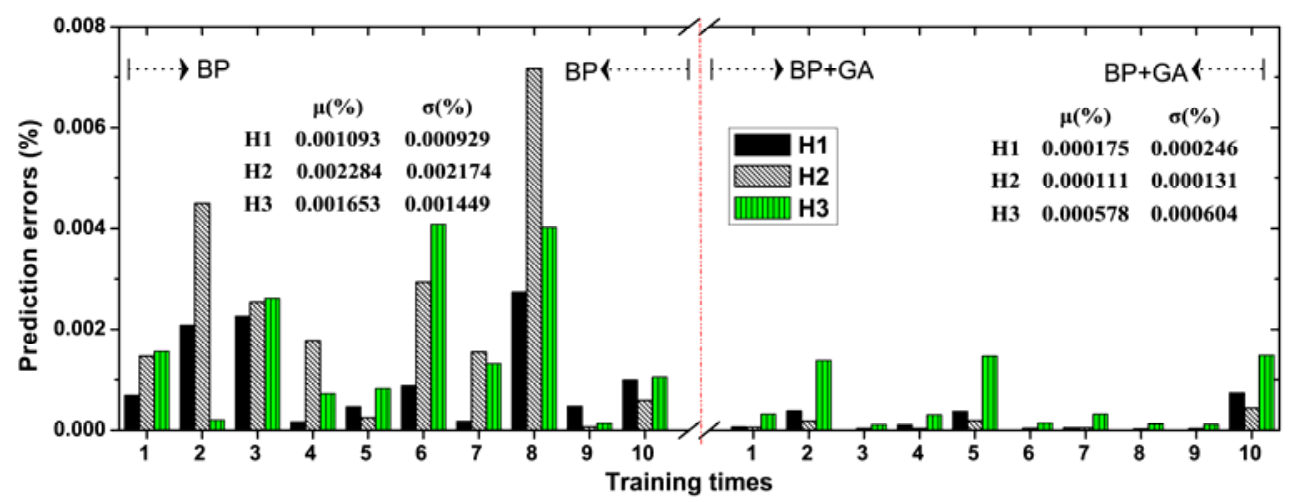

Figure 6. Prediction Errors of BP and BP + GA for Different Times

Figure 6 shows that the prediction errors are different even though the network structure and the training data are the same, especially for the prediction from BP without optimization by GA. The uniformity of the results is significantly improved after the initial weights and thresholds are optimized. The corresponding standard deviation $\sigma$ was reduced by $26.48 \%, 6.03 \%$ and $41.68 \%$ after optimization. The mean values $\mu$ of the prediction errors also decreased by $16.01 \%, 4.86 \%$ and $34.97 \%$. In general, the BP network optimized by GA became more accurate and stable for prediction. The optimization by GA, however, requires much more calculation time.

\section{CONCLUSIONS}

Based on this study, a method for the force finding of suspended-dome and some advice on the application of BP in the force finding can be obtained. The conclusions and suggestions are as follows:

(1) The construction process should be considered during the force finding of the suspended-dome because the appearance of pre-stress in the suspended-dome is related to it.

(2) The BP network can address the force finding of the suspended-dome, which is a problem category that cannot be formulated as an algorithm.

(3) A BP network can accurately predict the results of the force finding for the suspended-dome, and the prediction accuracy will become higher and higher while there is an increase in the training data size. Restricting the range of the training data according to the previous prediction results can also increase the prediction precision significantly. 
(4) Prediction results from the BP are different after repeated prediction regardless of its unchanged structure and training data because the initial weights and thresholds are generated randomly. After the initial weights and thresholds are optimized by the GA, the uniformity and the stability of the prediction results will be improved significantly. However, the optimization procedure will cost much more time.

(5) The BP network for force finding in this paper is a small-scale network. For a large-scale network, the determination of its network structure, especially the number of hidden layers and the node for each hidden layer, must be further studied.

\section{ACKNOWLEDGEMENTS}

The authors would like to acknowledge the financial support of the National Natural Science Foundation of China (No. 51108259; 51278461), the Science \& Technology Program of Shanghai Maritime University (No.20120050), the Research Innovation Projects of the Shanghai Municipal Education Commission (No.13YZ076), the Research Innovation Projects of 2013 Shanghai Postgraduate (20131129) and the Top Discipline Projects of the Shanghai Municipal Education Commission.

\section{REFERENCES}

[1] Gómez, J.V., "Tensegrity Structures and their Application to Architecture", MS thesis, School of Architecture, Queen's University Belfast, 2004.

[2] Pellegrino, S., "A Class of Tensegrity Domes", International Journal of Space Structures,1992, Vol. 7, No. 2, pp.127-143.

[3] Kawaguchi, M., Abe, M. and Hatato, T., "On a structural system "suspen-dome " System", Proceedings of IASS Symposium, Marid, Spain, 1993, pp. 523-530.

[4] Chen, Z.H., Wu, Y.J. and Yin, Y., et al, "Formulation and application of multi-node sliding cable element for the analysis of Suspen-Dome structures", Finite Elements in Analysis and Design, 2010, Vol. 46, No. 9, pp. 743-750.

[5] Kang, W.J., Chen, Z.H. and Lam, H.F., et al, "Analysis and Design of the General and Outmost-ring Stiffened Suspendome Structures”, Engineering Structures, 2003, Vol. 25, No. 13, pp. 1685-1695.

[6] Kawaguchi, M., Abe, M. and Tatemichi, I., "Design, Test, and Realization of "Suspend-Dome" System", Journal of IASS, 1999, Vol. 40, No. 131, pp. 179-192.

[7] Zhang, Z.H., Cao, Q.S. and Dong, S.L., "Structural Design of a Practical Suspendome", Advanced Steel Construction, 2008, Vol. 4, No. 4, pp. 323-340.

[8] Dong, S.L., Yuan, X.F. and Guo, J.M. et al, "Experimental Research on Tension Process of Suspen-dome Structural Model”, Spatial Structure, 2008, Vol. 14, No. 4, pp. 57-62.

[9] Tibert, A.G. and Pellegrino, S., "Review of Form-finding Methods for Tensegrity Structures", International Journal of Space Structures, 2003, Vol. 18, No. 4, pp. 209-223.

[10] Xu, X. and Luo, Y.Z., "Form Finding of Tensegrity Systems Using Simulated Annealing Algorithm", Journal of structural engineering, ASCE, 2010, Vol. 136, No. 8, pp. 1027-1031.

[11] Cao, Q.S. and Zhang, Z.H., "A Simplified Strategy for Force Finding Analysis of Suspendomes", Engineering Structures, 2010, Vol. 32, No. 1, pp. 306-318.

[12] Zhuo, X., Zhang, G.F. and Ishikawa, K., et al, "Tensile Force Correction Calculation Method for Prestressed Construction of Tension Structures", Journal of Zhejiang University, 2008, Vol. 9, No 9, pp. 1021-1027. 
[13] Guo, J.M., Dong, S.L. and Yuan, X.F., "Morphological Analysis of Suspend-dome Structures and Its Practical Analysis Method", China Civil Engineering Journal, 2009, Vol. 41, No. 12, pp.1-7. (in Chinese).

[14] Tsai, C.P., Lin, C. and Shen, J.N., "Neural Network for Wave Forecasting Among Multi-stations", Ocean Engineering, 2002, Vol. 29, No. 13, pp. 1683-1695.

[15] Xu, J.X., Zhao, J.C., and Wang, W.Z., et al., "Prediction of Temperature of Tubular Truss under Fire Using Artificial Neural Networks”, Fire. Safety Journal, 2013, Vol. 56, pp. 74-80.

[16] CECS 212-2006, "Technical Specification for Pre-stressed Steel Structure" China Plan Press, Beijing, 2006. (in Chinese).

[17] Kriesel, D., "Neural Networks", Dkriesel.com, 2005.

[18] Gao, D.Q., "On Structures of Supervised Linear Basis Function Feed Forward Three-layer Neural Networks", Chinese Journal Computer, 1998, Vol. 21, No. 1, pp. 80-86. (in Chinese). 